Revue internationale P.M.E.

Économie et gestion de la petite et moyenne entreprise

\title{
Relations entre l'exportation, le développement organisationnel et la situation financière des PME canadiennes
}

\section{Josée St-Pierre}

Volume 16, numéro 2, 2003

URI : https://id.erudit.org/iderudit/1008439ar

DOI : https://doi.org/10.7202/1008439ar

Aller au sommaire du numéro

Éditeur(s)

Presses de l’Université du Québec

ISSN

0776-5436 (imprimé)

1918-9699 (numérique)

Découvrir la revue

Citer cet article

St-Pierre, J. (2003). Relations entre l'exportation, le développement organisationnel et la situation financière des PME canadiennes. Revue internationale P.M.E., 16(2), 61-82. https://doi.org/10.7202/1008439ar

\section{Résumé de l'article}

L'exportation des PME est devenue un sujet de préoccupation de plus en plus important pour différents gouvernements qui y voient la possibilité de réduire leur déficit commercial tout en dynamisant le développement économique régional. Malgré l'intérêt que cela présente, un pourcentage relativement faible de PME exportent une partie substantielle de leur production. Évidemment, ce ne sont pas toutes les PME qui ont le potentiel d'exporter. Mais parmi celles qui l'ont, on se demande pourquoi une proportion limitée acheminent vers d'autres pays leurs produits/services. Est-ce parce que leur marché national est suffisant, que les entrepreneurs sont réticents et appréhendent ces activités extérieures, ou est-ce que l'exportation a des conséquences financières difficiles à gérer pour les entrepreneurs ? C'est ce dernier élément qui nous intéresse dans cette étude, où nous analysons les caractéristiques financières et organisationnelles d'un groupe de PME exportatrices, que nous comparons à d'autres PME qui ont décidé de concentrer leurs activités sur le marché intérieur. Parmi les résultats obtenus, nous retenons que la taille des entreprises est liée à la probabilité d'exporter, à la diversité et à la destination des exportations. Les PME exportatrices sont plus rentables que les autres, mais elles sont également plus innovatrices et plus souvent engagées dans diverses collaborations d'affaires. L'innovation des PME est un facteur clé et le premier facteur explicatif de l'exportation et du succès de l'entreprise sur les marchés étrangers.
Ce document est protégé par la loi sur le droit d'auteur. L'utilisation des services d'Érudit (y compris la reproduction) est assujettie à sa politique d'utilisation que vous pouvez consulter en ligne.

https://apropos.erudit.org/fr/usagers/politique-dutilisation/ 


\title{
Relations entre l'exportation, le développement organisationnel et la situation financière des PME canadiennes
}

Josée ST-PIERRE'

Université du Québec à Trois-Rivières

MOTS CLÉS

PME - Exportation - Développement de marché

Performance financière - Rentabilité

\begin{abstract}
RÉSUMÉ
L'exportation des PME est devenue un sujet de préoccupation de plus en plus important pour différents gouvernements qui y voient la possibilité de réduire leur déficit commercial tout en dynamisant le développement économique régional. Malgré l'intérêt que cela présente, un pourcentage relativement faible de PME
\end{abstract}

\section{L'AUTEURE}

JosÉE ST-PIERRE est détentrice d'un doctorat en finance et professeure titulaire en finance au Département des sciences de la gestion de l'Université du Québec à Trois-Rivières. Elle dirige le Laboratoire de recherche sur la performance des entreprises (http://www.uqtr.ca/inrpme/ larepe) dont la mission est de mieux comprendre les déterminants et les mesures de performance chez les PME ainsi que les facteurs d'échec; elle est également titulaire de la Chaire J.-Armand Bombardier sur les relations interentreprises et la gestion du risque. Elle est responsable du développement des outils de benchmarking pour les PME PDG ${ }^{\circledR}$ et Balise, et du logiciel d'évaluation du risque des PME eRisC. Outre les aspects liés au financement, ses intérêts de recherche portent essentiellement sur les mesures et les déterminants de la performance et du risque des PME, domaine dans lequel elle a réalisé de nombreuses recherches. Adresse : Université du Québec à Trois-Rivières, C.P. 500, Trois-Rivières, Québec, Canada, G9A 5H7. Téléphone: (819) 376-5011 poste 4052. Télécopieur: (819) 376-5138. Courriel : <josee.st-pierre@dr.cgocable.ca>.

1. L'auteure aimerait remercier les évaluateurs de la revue pour la pertinence de leurs commentaires, Pierre-André Julien pour l'ajout de certaines notes importantes et Daniel Pitre pour la réalisation du travail statistique. Les idées et conclusions demeurent l'entière responsabilité de l'auteure. 
exportent une partie substantielle de leur production. Évidemment, ce ne sont pas toutes les PME qui ont le potentiel d'exporter. Mais parmi celles qui l'ont, on se demande pourquoi une proportion limitée acheminent vers d'autres pays leurs produits/services. Est-ce parce que leur marché national est suffisant, que les entrepreneurs sont réticents et appréhendent ces activités extérieures, ou est-ce que l'exportation a des conséquences financières difficiles à gérer pour les entrepreneurs? C'est ce dernier élément qui nous intéresse dans cette étude, où nous analysons les caractéristiques financières et organisationnelles d'un groupe de PME exportatrices, que nous comparons à d'autres PME qui ont décidé de concentrer leurs activités sur le marché intérieur. Parmi les résultats obtenus, nous retenons que la taille des entreprises est liée à la probabilité d'exporter, à la diversité et à la destination des exportations. Les PME exportatrices sont plus rentables que les autres, mais elles sont également plus innovatrices et plus souvent engagées dans diverses collaborations d'affaires. L'innovation des PME est un facteur clé et le premier facteur explicatif de l'exportation et du succès de l'entreprise sur les marchés étrangers.

\begin{abstract}
Exports by small and medium-sized enterprises are a growing concern for various governments who see them as a means of reducing their trade deficit while, at the same time, stimulating regional economic development. Despite the interest SMEs generate, only a relatively small percentage of them exports a substantial portion of their goods and services. It is evident that not all of them have export potential. This raises the question as to why only a limited number of those that do have the potential do indeed export. Is it because domestic markets are sufficient? Are entrepreneurs reluctant because they are wary of doing business outside their own market? Does exporting present financial hurdles that are too difficult for them to tackle? This last question is the focus of this article devoted to a comparative study of the financial and organizational characteristics of a group of SMEs which export, and those of SMEs which concentrate their activities in the domestic market. Findings reveal that business size is a factor linked to export potential as well as to the diversity and destination of exports. Exporting SMEs are more profitable than others; they are also more innovative and are more often involved in various business collaborations. Innovation in SMEs is a critical factor; it is the key to exports, and to success on the foreign market.
\end{abstract}

\title{
RESUMEN
}

La exportación de las PyMEs se ha convertido en un tema de preocupación cada vez más importante para los diferentes gobiernos, quienes ven con eso la posibilidad de reducir su déficit comercial, dinamizando al mismo tiempo el desarrollo económico regional. A pesar del interés que esto representa, un porcentaje relativamente débil de PyMEs exportan una parte sustancial de su producción. Evidentemente, no todas las PyMEs tienen el potencial de exportar. Pero entre aquellas que lo tienen, uno se pregunta por qué una proporción limitada orientan hacia otros países sus productos o servicios. ¿Es porque su mercado nacional es suficiente, porque los empresarios son reticentes y temen estas actividades con el exterior, o la exportación entraña consecuencias financieras difíciles de manejar

Revue internationale P.M.E., vol. 16, $\mathrm{n}^{\circ}$ 2, 2003

(C) 2003 - Presses de l'Université du Québec

Édifice Le Delta I, 2875, boul. Laurier, bureau 450, Sainte-Foy, Québec G1V 2M2 • Tél. : (418) 657-4399 - www.puq.uquebec.ca

Tiré de : Revue internationale P.M.E., vol. $16, \mathrm{n}^{\circ} 2$, sous la direction de Pierre-André Julien. 
por los empresarios ? Es este último elemento que nos interesa en este estudio, donde analizaremos las características financieras y organizacionales de un grupo de PyMEs exportadoras, que comparamos con otras PyMEs que han decidido concentrar sus actividades en el mercado interno. Entre los resultados obtenidos, se tiene que el tamaño de las empresas está relacionado con la probabilidad de exportar, con la diversidad y con el destino de las exportaciones. Las PyMEs exportadoras son más rentables que las otras, pero ellas son igualmente más innovadoras y la mayoría de las veces involucradas en diversas colaboraciones de negocios. La innovación de las PyMEs es un factor clave importante y el primer factor explicativo de la exportación y del éxito de la empresa en los mercados externos.

\section{ZUSAMMENFASSUNG}

Der Export von KMU ist ein immer wichtigeres Anliegen für verschiedene Regierungen geworden, die eine Möglichkeit sehen, ihr Handelsdefizit zu reduzieren, indem sie die regionale Wirtschaftsentwicklung dynamisieren. Trotz diesem Interesse, exportiert nur ein ziemlich schwacher Prozentsatz der KMU einen wesentlichen Teil ihrer Produktion. Selbstverständlich sind es nicht alle KMU, die das Potential haben zu exportieren. Aber unter jenen, die das Potential haben, fragt man sich, warum nur ein begrenzter Anteil der Betriebe ihre Produkte/Dienste in andere Länder exportiert. Ist der Umstand des ausreichenden nationalen Marktes der Grund für die passive Haltung gegenüber den Außenaktivitäten ? Oder bewirkt der Export für die Unternehmen schwierig zu verwaltende finanzielle Folgen? Es ist dieses letzte Element, das uns in dieser Studie interessiert, wo wir die finanziellen und organisatorischen Eigenschaften einer Gruppe von exportierenden KMU analysieren. Wir vergleichen diese Gruppe mit anderen kleinen und mittleren Unternehmen, die beschlossen haben, ihre Aktivitäten auf den Binnenmarkt zu konzentrieren. Unter den erzielten Ergebnissen können wir festhalten, daß die Größe der Unternehmen im Zusammenhang steht mit der Wahrscheinlichkeit des vielfältigen Exportes in unterschiedliche Destinationen. Die exportierenden KMU sind rentabler als die auf den Binnenmarkt fokussierten Betriebe, und sind gleichzeitig innovativer und öfters in verschiedentliche Geschäftsbeziehungen integriert. Die Innovation der KMU ist ein wichtiger Schlüsselfaktor und der erste erklärende Faktor für den Export und den Erfolg des Unternehmens auf den ausländischen Märkten.

\section{Introduction}

Les ventes à l'étranger posent habituellement plusieurs difficultés que les entreprises n'ont pas à surmonter sur le plan local. Des coûts de production et de préparation plus élevés, des délais plus longs, une incertitude plus grande (Léonidou et AdamsFlorou, 1997), voilà quelques raisons qui expliquent que l'exportation ne soit pas une voie de développement empruntée par la plupart des entreprises de petite et moyenne tailles qui auraient le potentiel de le faire. De plus, comme le souligne Léo (2000, p. 47), «[...] se développer à l'international, même simplement par 
l'exportation, constitue pour des petites firmes, un saut qualitatif important qui remet en cause nombre de procédures, d'habitudes et de structures mêmes qui avaient fait leurs preuves sur le marché strictement local ou national ». De Toni et Nassimbeni (2001) ajoutent que les PME font face à trois obstacles dans le développement de leurs activités vers l'exportation : le manque d'information concernant les marchés étrangers, l'accès à du financement adéquat et des procédures administratives plus complexes que celles nécessaires sur les marchés intérieurs.

Malgré tout, plusieurs gouvernements voient dans l'exportation une clé pour le développement économique de leurs régions, puisque les marchés intérieurs ne peuvent croître indéfiniment et permettre une expansion économique soutenue. De plus, la stimulation de l'exportation est reconnue par certains gouvernements comme une façon de réduire le déficit de la balance commerciale et de dynamiser les activités économiques des entreprises locales (Westhead, 1995; Mehran et Moini, 1999; Wolff et Pett, 2000). La mise sur pied de divers comités d'étude sur le sujet, la création de plusieurs programmes de formation et de soutien financier spécifiques pour les entreprises exportatrices ou désireuses de le devenir sont des indicateurs concrets de l'intérêt des gouvernements dans le développement des activités d'exportation chez les $\mathrm{PME}^{2}$. Par ailleurs, ces mêmes gouvernements affichent leur méconnaissance du fonctionnement des PME face à l'exportation et des conséquences de celle-ci sur leur développement, leur risque et leur rentabilité (Bijmolt et Zwart, 1994).

Au Canada, la part des échanges internationaux est passée de $28 \%$ du PIB en 1984 à plus de $38 \%$ en $1996^{3}$. Différentes estimations indiquent cependant que seulement 8,5\% des PME canadiennes exportent, et que leur degré d'exportation est variable mais relativement faible. Évidemment, ce ne sont pas toutes les PME qui ont la possibilité d'exporter leurs produits/services, mais, parmi celles qui peuvent le faire, il est intéressant de comprendre pourquoi une grande proportion se concentre sur leur marché national. Est-ce que les exigences, les difficultés et la complexité des ventes au-delà des frontières peuvent avoir des conséquences financières telles, que les entreprises de petite dimension ne présentant pas un profil particulier seraient moins susceptibles d'exporter que d'autres? Dans l'état actuel des connaissances, il est difficile de répondre à cette question, puisque la littérature sur l'exportation chez les PME n'aborde que de façon très marginale les dimensions financières.

2. Signalons, à ce sujet, que l'origine de cette étude est directement liée à l'octroi d'un mandat de recherche d'un organisme gouvernemental de développement économique et industriel, désireux de savoir pourquoi peu de PME exportent et si des programmes de financement particuliers pourraient changer quoi que ce soit à cette situation.

3. Statistique Canada, 1994-1996.

Revue internationale P.M.E., vol. 16, $\mathrm{n}^{\circ}$ 2, 2003 
C'est ce dont nous discutons dans cette étude qui se veut surtout exploratoire, où nous comparons les informations organisationnelles et financières de PME ayant des activités d'exportation développées, à celles de PME qui se concentrent sur les marchés locaux. Nous voulons ainsi vérifier s'il existe des différences qui pourraient, éventuellement, permettre de mieux comprendre ce qui motive certaines PME à exporter leurs produits / services.

\section{L'exportation chez les PME}

\subsection{Relation entre la taille des entreprises et les activités internationales}

Un rapport de l'OCDE (cité dans Reynolds, 1997) révèle qu'environ $10 \%$ des PME de 18 pays membres, principalement des PME manufacturières, sont impliquées dans des investissements internationaux directs.

Au Canada, les statistiques sont différentes et certaines estimations montrent que seulement 8,5\% des PME canadiennes exportaient en 1995 (Industry Canada, 1997). Ces statistiques cachent toutefois une réalité qu'il importe de considérer. Ce ne sont pas toutes les $\boldsymbol{P M E}$ qui ont le potentiel d'exporter étant donné les caractéristiques de leurs produits / services, de même que leur degré de développement organisationnel (75\% des PME canadiennes ont moins de cinq employés). Par ailleurs, l'exportation n'est pas la seule et unique voie pour assurer la croissance des entreprises. Avant de vendre à l'étranger ses biens / services, l'entreprise tentera probablement de saturer le marché intérieur, ce qui peut parfois être largement suffisant pour assurer plusieurs années de croissance.

Quant à la part de leur production qui est exportée, les résultats d'un sondage de la Banque de développement du Canada (1997) ${ }^{4}$ auprès de 547 dirigeants de PME innovantes indiquent que les exportations de ces compagnies pouvaient représenter jusqu'à $25 \%$ de leur chiffre d'affaires. Au Canada, la FCEI, dans un sondage auprès de 8141 PME de divers secteurs, a également observé une relation positive entre la taille de l'entreprise (mesurée en nombre d'employés) et le degré d'exportation.

Ces statistiques, ainsi que celles produites par la Bank of England (1998), font ressortir une relation entre la taille de l'entreprise et sa propension à vendre ses produits à l'étranger. Cette relation entre la taille peut cependant ne pas être linéaire et Julien (2000) suppose plutôt l'existence d'un seuil (aux environs de 40 employés chez les entreprises manufacturières) en deçà duquel il est peu probable de trouver des exportateurs « organisés ».

4. Une enquête de la Fédération canadienne de l'entreprise indépendante (FCEI) produit les mêmes résultats.

Revue internationale P.M.E., vol. 16, nº 2, 2003 
L'hypothèse d'une relation entre la taille de l'entreprise et l'exportation pourrait s'inscrire dans la théorie des stades de développement. Selon cette théorie, l'accès aux marchés internationaux se fait selon un cheminement progressif, où l'entreprise développe d'abord son expertise et maîtrise ses façons de faire avant de s'engager dans de nouvelles activités où l'incertitude est plus grande (Wolff et Pett, 2000). À ce sujet, Westhead (1995) relève que les PME exportatrices sont significativement plus grandes que les PME non exportatrices alors que Cavusgil (1984) trouve une relation positive entre la taille de la firme et l'intensité d'exportation. Cependant, ce dernier résultat n'est pas confirmé par Bonaccorsi (1992) qui affirme que la probabilité d'être un exportateur augmente avec la taille de la firme, mais que celle-ci n'a aucun effet significatif sur le degré d'exportation. Il soutient par ailleurs que les entreprises peuvent exporter grâce à leur participation à des réseaux externes pour distribuer leurs ressources, ce qui atténuerait le rôle joué par la taille.

Par ailleurs, Walters et Samiee (1990) stipulent que la petite taille semble contraindre l'étendue des opérations d'exportation de la firme, favorisant une activité internationale limitée en termes de diversité de produit-marché. À cet égard, Calof (1993) note l'effet de la taille de la firme sur la limitation du nombre de marchés d'exportation. De son côté, Sharmistha (1999) observe également une relation positive entre la taille des PME et l'exportation qu'il nuance en affirmant que cette relation est moins évidente pour les firmes qui exportent plus activement. Ses observations laissent entrevoir l'existence d'autres facteurs qui pourraient influencer le degré d'exportation des PME, comme l'étendue du marché local, l'organisation de l'entreprise, les caractéristiques de son produit/ service et, évidemment, la volonté de l'entrepreneur d'étendre ses activités en dehors de ses frontières.

En outre, Lefebvre, Lefebvre et Bourgault (1998) soutiennent que la taille de la firme semble constituer un obstacle pour la pénétration des marchés de proximité, mais elle n'a aucun effet sur sa capacité à exporter sur les marchés éloignés. La principale difficulté réside vraisemblablement dans la mobilisation des ressources nécessaires pour les premières étapes d'internationalisation (pénétration des marchés de proximité). Cette difficulté s'amoindrit dans les étapes suivantes du processus d'internationalisation, lorsque les firmes commencent à exporter sur les marchés plus éloignés.

La taille pourrait être un frein à l'exportation des petites entreprises étant donné que ces dernières n'ont pas toujours les ressources suffisantes pour procéder à des activités qui peuvent parfois être complexes et nécessiter des modifications à leurs façons de faire, l'exportation créant des besoins spécifiques, comme nous le verrons dans les paragraphes suivants. 


\subsection{Les besoins financiers liés à l'exportation}

La production des biens pour les marchés étrangers est souvent plus coûteuse que pour les marchés locaux parce qu'elle nécessite habituellement des précautions particulières et le respect de certaines normes ou standards, étant donné des différences culturelles, linguistiques et réglementaires entre les pays importateur et exportateur (Lee et Jang, 1998; Clarke, 2000). Par ailleurs, étant donné la complexité des ventes à l'étranger et la concurrence à laquelle les PME devront faire face, De Toni et Nassimbeni (2001) signalent que, pour réussir l'exportation, les entreprises doivent faire appel à certaines pratiques de gestion de la production avancées, comme le juste à temps, la qualité totale ou l'ingénierie simultanée. Ces besoins particuliers devraient entraîner des coûts supplémentaires par rapport à ceux que requiert le marché local.

D'un autre côté, la distance séparant l'importateur et l'exportateur occasionne des délais plus importants dans le transfert des marchandises et accroît les difficultés d'évaluation de la qualité de paiement du client étranger; cela peut influer sur les périodes de récupération des comptes ainsi que sur le risque d'être impayé. Cette situation est prise en compte par les institutions financières qui vont réduire leur offre de financement aux PME exportatrices, étant donné le risque associé aux actifs à court terme internationaux tels les comptes clients et les stocks, qui auront une valeur de garantie inférieure à celle des mêmes actifs destinés à des marchés intérieurs. Cela oblige alors les PME à autofinancer en grande partie leurs besoins en fonds de roulement (Cooper et Nyborg, 1998). Évidemment, l'entreprise peut avoir recours à tout un ensemble de documents du commerce international pour réduire ces inconvénients et sécuriser les transactions, mais ils sont relativement coûteux et peu utilisés par les PME, ce qui est confirmé par les résultats de l'étude de Samuels, Greenfield et Mpuku (1992). Ces auteurs observent que la lettre de crédit, document clé en commerce international pour le règlement des comptes, ainsi que les avances de paiement sont marginalement utilisés par les PME exportatrices (moins de $30 \%$ des cas dans les pays industrialisés).

Les besoins financiers liés aux transactions internationales sont ainsi plus importants que ceux inhérents aux transactions nationales. L'augmentation des délais dans la récupération des sommes engagées dans les activités d'exportation ainsi que certaines spécifications particulières au niveau de la production accroissent sensiblement les besoins en liquidités, en fonds de roulement et en système de production.

Ces besoins financiers plus grands pourraient expliquer pourquoi relativement peu de PME exportent leurs produits et, lorsqu'elles le font, pourquoi elles exportent habituellement une faible partie de leur production, comme nous l'avons mentionné plus haut.

Revue internationale P.M.E., vol. 16, $\mathrm{n}^{\circ}$ 2, 2003

(C) 2003 - Presses de l'Université du Québec 
L'ensemble de ces considérations permet de supposer que l'exportation influence de façon directe ou indirecte les résultats financiers des PME. La littérature financière ne nous offre cependant pas beaucoup de preuves de ces effets, alors que leur connaissance permettrait peut-être de faciliter le développement de cette activité pouvant contribuer à la performance des PME, des institutions financières qui leur viendraient en appui et, finalement, des gouvernements qui pourraient compter davantage sur celle-ci pour assurer une croissance économique soutenue.

\section{Hypothèses de recherche et méthodologie}

D'après la recension de la documentation, peu d'auteurs se sont intéressés aux particularités financières des PME qui œuvrent sur les marchés internationaux. Pourtant, l'intérêt de tels résultats est manifeste, autant pour les bailleurs de fonds qui doivent mieux comprendre les particularités des PME qu'elles financent, pour les autorités gouvernementales qui veulent assurer un soutien plus substantiel pour favoriser les activités internationales, que pour les entreprises elles-mêmes qui pourraient ainsi mieux comprendre les effets de l'exportation sur leur situation financière.

\subsection{Hypothèses}

La littérature présentée plus haut nous permet de formuler quelques hypothèses afin de mieux saisir les relations entre l'exportation et la situation financière des PME.

Sharmistha (1999), dans une étude menée auprès de 54 PME ontariennes visant à examiner les caractéristiques des entreprises qui exportent de façon plus régulière et en quantité plus importante ainsi que celles qui exportent plutôt de façon ad hoc, note que les firmes ayant une forte orientation vers l'exportation accordent plus d'importance au développement de nouveaux produits sur une base continue ainsi qu'à l'amélioration des méthodes de production existantes. Julien (2000) ajoute à cela qu'il faut remplir au moins deux conditions pour réussir à l'exportation : avoir un marché de base national solide et offrir des produits relativement originaux et innovateurs ${ }^{5}$. Cette affirmation est appuyée par De Toni et Nassimbeni (2001) qui soutiennent que la complexité des activités internationales

5. Il peut toutefois exister des entreprises qui exportent une grande partie de leur production qui est très spécialisée, sur des marchés bien définis et connus, alors que leur marché national est peu développé. Cette hypothèse qui exige une connaissance précise des marchés et des produits des entreprises exportatrices ne pourra cependant pas être vérifiée dans cette étude, ce qui en constituera une des limites. 
exige des PME qu'elles aient une structure organisationnelle bien développée, qu'elles utilisent les pratiques de gestion avancées et que leur système de production soit relativement sophistiqué afin de satisfaire aux exigences des importateurs. Ces affirmations pourraient nous mener à l'hypothèse suivante :

\section{$H_{1}$ : Les PME exportatrices ont un développement organisationnel diffé- rent des autres (pratiques de gestion, activités de $R$-D, système de production, etc.).}

Par ailleurs, étant donné les ressources limitées des PME, on pourrait s'attendre à ce que celles qui connaissent du succès à l'exportation (mesuré selon le volume d'exportation) affichent une productivité supérieure aux autres, étant donné les exigences de qualité et de compétitivité auxquelles elles doivent satisfaire sur la scène internationale.

\section{$H_{2}$ : Les PME exportatrices sont plus productives que les autres.}

Miller (1994, cité dans Zahra, Neubaum et Huse, 1997) affirme que la technologie est rapidement devenue une source majeure d'avantage compétitif sur les marchés internationaux. À cet effet, Walters et Samiee (1990) trouvent que le produit de haute technologie exporté est positivement associé à la rentabilité de l'exportation. Ce résultat vient appuyer les études qui ont souligné l'importance de l'intensité technologique du produit comme facteur de succès à l'exportation (Cavusgil et Nevin, 1981 ; Cooper et Kleinschmidt, 1985) ${ }^{6}$. Dans ce sens, les études de Sharmistha (1999), Julien (1993) et Beamish et Munro (1986) indiquent que les PME qui exportent des produits ayant une forte valeur ajoutée ont de meilleures chances de percer les marchés étrangers. En revanche, rappelons que l'exportation entraîne des coûts de production plus élevés étant donné divers éléments spécifiques tels que l'emballage, le transport et l'adaptation de la production. Dans ces conditions, les coûts de production et de préparation plus élevés, ainsi que ceux imputables aux activités de R-D seraient absorbés par des prix de vente supérieurs et les marges de profit entre les exportateurs et les non-exportateurs devraient être identiques. Voici ainsi notre troisième hypothèse :

\section{$H_{3}$ : Les PME exportatrices sont aussi rentables que les autres.}

Finalement, la diversité des exportations devrait influencer les résultats financiers des PME. Beamish, Craig et McLelland (1993) affirment que le nombre de marchés dans lesquels chaque entreprise distribue ses produits est lié positivement au succès de l'exportation. L'étendue des exportations peut être le résultat de l'évolution des activités internationales de l'entreprise, celle-ci profitant de son succès sur son premier marché pour étendre son expérience en visant un plus grand

6. Cités dans Walters et Samiee (1990).

Revue internationale P.M.E., vol. 16, $\mathrm{n}^{\circ}$ 2, 2003 
nombre de pays. En outre, on peut supposer que les firmes qui ont connu des échecs sur leur premier marché d'exportation ne chercheront pas à étendre leurs activités. D'ailleurs, le nombre de pays vers lesquels la firme exporte ses produits a été utilisé par plusieurs auteurs comme un indicateur du succès de l'exportation (Calof, 1993 ; Sriram et Manu, 1995)7 . De plus, la présence sur différents marchés évoluant à des rythmes non synchronisés permet à l'exportateur de diversifier les risques liés aux fluctuations des devises et aux changements politiques dans les pays étrangers (Dalli, 1995, cité dans Stump, Athaide et Axinn, 1998) et d'accroître sa performance.

\section{$H_{4}$ : La rentabilité des PME exportatrices est influencée par le nombre de marchés d'exportation dans lesquels elles distribuent leurs produits.}

\subsection{Description de l'échantillon et tests statistiques utilisés}

Pour vérifier nos hypothèses de recherche, nous avons utilisé la base de données $\mathrm{PDG}^{\circledR 8}$, qui contient des informations sur 323 PME manufacturières canadiennes comptant entre 20 et 400 employés. On y trouve plus de 850 variables générales et financières qui ont été recueillies directement auprès d'entreprises manufacturières qui avaient à remplir un questionnaire d'informations confidentielles. Les entreprises sont contactées directement pour fournir leurs informations générales et financières en échange d'un diagnostic sur leur situation générale.

Ce processus de recueil d'informations assure une grande fiabilité à la base de données utilisée qui réunit des informations sur les sujets suivants, obtenues pour la dernière année d'activité : le profil de l'entrepreneur et son orientation quant au développement de son entreprise; les divers éléments de la gestion des ressources humaines tels que la formation, les programmes de rémunération, les pratiques d'évaluation de rendement et le climat de travail ; les informations technologiques sur le type de production de l'entreprise, les équipements et les systèmes qu'elle utilise, les investissements dans les nouvelles technologies, les activités de R-D, sur les équipements et les procédés de fabrication, etc. ; les activités de développement de marché et de service à la clientèle ; les conditions de crédit bancaire et le degré de satisfaction de l'entrepreneur et, finalement, les résultats financiers pour les cinq derniers exercices.

7. Cités dans Zahra et al. (1997).

8. L'auteure aimerait remercier Développement Économique Canada et le Groupement des chefs d'entreprise du Québec qui, conjointement avec le Laboratoire de recherche sur la performance des entreprises, ont permis la création de cette base de données.

Revue internationale P.M.E., vol. 16, $\mathrm{n}^{\circ}$ 2, 2003

(C) 2003 - Presses de l'Université du Québec 
Les informations recueillies auprès des entreprises ne l'ont pas été pour une étude sur l'exportation comme telle, mais afin de constituer une base de données de tous les éléments ayant une influence directe ou indirecte sur la performance ou les difficultés d'une entreprise manufacturière. Certaines variables pertinentes seront ainsi absentes de notre étude. Par ailleurs, celle-ci sera enrichie par l'analyse d'un ensemble de variables générales que l'on trouve rarement dans les études très spécialisées. Finalement, étant donné que l'exportation sera mesurée simplement par la distribution du chiffre d'affaires lors de la dernière année d'exploitation, nous ne pourrons établir de relations causales entre les résultats financiers et l'exportation des PME.

Soulignons que l'échantillon étudié est particulier et ne sera pas représentatif de l'ensemble de la population, étant donné que les entreprises sollicitées font partie, pour la plupart, de regroupements d'entreprises ou de gens d'affaires, qu'elles échangent souvent entre elles beaucoup d'informations favorisant leur développement et qu'elles participent toutes à une activité de «benchmarking » compétitif. Elles n'ont donc pas les caractéristiques des PME isolées comme on les voit souvent.

TABLEAU 1

Quelques caractéristiques sommaires de l'échantillon

\begin{tabular}{lccc}
\hline $\mathrm{N}=323$ & Écart type & Médiane & Moyenne \\
\hline Chiffre d'affaires & $8,9 \mathrm{M} \$$ & $4,9 \mathrm{M} \$$ & $7,6 \mathrm{M} \$$ \\
Nombre d'employés & 56,8 & 44,0 & 60,8 \\
Croissance annuelle des ventes & & & \\
$\quad$ des trois dernières années & $32,6 \%$ & $14,3 \%$ & $21,5 \%$ \\
Âge des entreprises & 19,0 & 21,0 & 25,4 \\
Taux d'exportation & $27,1 \%$ & $10,0 \%$ & $20,9 \%$ \\
Pourcentage des ventes consacré à la R-D & $4,5 \%$ & $0,72 \%$ & $2,19 \%$ \\
\hline
\end{tabular}

Compte tenu du caractère exploratoire de cette étude et de l'absence de relations causales, nous utiliserons des tests statistiques univariés pour vérifier nos hypothèses de recherche. Nous recourrons à des tests non paramétriques pour les différences de médiane ou de moyenne, selon leur distribution ${ }^{9}$, dans le cas des variables réelles et métriques et des tests de chi carré pour les variables discrètes. Le logiciel statistique SAS a été employé pour vérifier les hypothèses.

9. Notons que si le test statistique est réalisé sur des moyennes plutôt que sur des médianes, le seuil critique de reconnaissance des écarts significatifs sera différent, comme nous le verrons aux tableaux 2,3 et 7 .

Revue internationale P.M.E., vol. 16, $\mathrm{n}^{\circ}$ 2, 2003 


\section{Présentation et analyse des résultats}

Notre première hypothèse suppose que les PME exportatrices ont un développement organisationnel différent des autres. Pour vérifier cette hypothèse, nous avons considéré le développement organisationnel d'abord du côté des activités de gestion et ensuite du côté des activités de production ; ces résultats sont présentés au tableau 2.

Les résultats confirment ceux obtenus par d'autres auteurs notamment en ce qui concerne la relation entre la taille et l'exportation (Westhead, 1995; Wolf et Pett, 2000). On constate effectivement que les entreprises exportatrices sont significativement plus grandes que les autres, que la taille soit mesurée en volume d'activités ou en nombre d'employés. En revanche, les entreprises n'ont pas connu des taux de croissance différents dans les trois dernières années, contredisant ainsi l'affirmation voulant que l'exportation soit le résultat d'une croissance soutenue et qui ne peut être assurée sur le marché local ou national (Westhead, 1995).

Contrairement aux résultats de Moini (1995), on ne trouve pas un plus grand degré d'expertise chez les entreprises exportatrices, lorsque celle-ci est mesurée par la présence d'un responsable pour les fonctions finance et marketing, et on relève une moins grande formalisation de la structure organisationnelle d'après le rapport du nombre d'employés au nombre de responsables présents dans l'organisation. Ce dernier résultat pourrait être lié au comportement particulier des PME innovantes qui, comme l'avance Heunks (1998), ont besoin d'un environnement créatif et peu formalisé pour accroître leur taux d'innovation. Or, comme les PME exportatrices sont également fortement engagées dans des activités de R-D, on pourrait supposer qu'un nombre plus élevé d'employés sous la responsabilité d'un chef de département serait un indicateur de flexibilité.

Ces résultats sont également contraires aux propositions de DeToni et Nassimbeni (2001), quant au niveau d'organisation que l'on suppose plus important chez les entreprises exportatrices ; on trouvera les mêmes conclusions pour la structure de production. D'après ces auteurs, pour pouvoir connaître du succès à l'exportation, les entreprises doivent avoir développé le concept de qualité et posséder une structure technologique relativement sophistiquée. Nos résultats ne mettent pas en évidence ces hypothèses, qui n'avaient d'ailleurs pas été confirmées par ces auteurs ${ }^{10}$. Toutefois, le fait que les exportateurs soient plus souvent engagés dans des collaborations d'affaires pourrait expliquer qu'ils ne présentent pas un développement organisationnel différent des autres, celui-ci ayant pu être sous-traité à un «collaborateur».

10. Rappelons toutefois les particularités de notre échantillon et le fait que les PME étudiées s'adonnent toutes à la pratique du «benchmarking», ce qui, en soit, est indicatif d'un type de PME plutôt orienté vers la qualité et l'amélioration continue (Matheson, 2000).

Revue internationale P.M.E., vol. 16, $\mathrm{n}^{\circ}$ 2, 2003 
TABLEAU 2

Relations entre l'exportation et le degré de développement organisationnel des entreprises

\begin{tabular}{|c|c|c|c|}
\hline & $\begin{array}{l}\text { Exportateurs } \\
\quad(\mathrm{N}=237)\end{array}$ & $\begin{array}{l}\text { Non- } \\
\text { exportateurs } \\
(\mathrm{N}=86)\end{array}$ & $\begin{array}{c}\text { Test } \\
\text { statistique } \\
\text { (Probabilité) }\end{array}$ \\
\hline $\begin{array}{l}\text { Chiffre d'affaires à la dernière année } \\
\text { d'exploitation }\end{array}$ & $5,4 \mathrm{M} \$$ & $3,5 \mathrm{M} \$$ & $2,22 * *$ \\
\hline Nombre d'employés & 48 & 35 & $3,01 * * *$ \\
\hline Âge des entreprises & 21,5 & 20,0 & 0,53 \\
\hline $\begin{array}{l}\text { Taux de croissance des ventes } \\
\text { des trois dernières années }(\%)\end{array}$ & 15,08 & 12,39 & 1,04 \\
\hline \multicolumn{4}{|l|}{ Développement organisationnel des entreprises } \\
\hline $\begin{array}{l}\text { Pourcentage d'entreprises ayant un responsable } \\
\text { finance et/ou marketing }\end{array}$ & 97,89 & 95,35 & 1,50 \\
\hline $\begin{array}{l}\text { Nombre d'employés / nombre de responsables } \\
\text { de service }\end{array}$ & 11,2 & 8,16 & $2,00 * *$ \\
\hline Budget de formation / nombre d'employés (\$) & 350,81 & 318,29 & 0,73 \\
\hline $\begin{array}{l}\text { Pourcentage d'entreprises utilisant } \\
\text { la gestion participative }\end{array}$ & 54,66 & 64,29 & 2,35 \\
\hline Budget de R-D (\% des ventes) & 2,56 & 0,95 & $8,77 * * *$ \\
\hline Pourcentage d'entreprises ayant un produit maison & 57,81 & 45,35 & $3,97 * *$ \\
\hline $\begin{array}{l}\text { Pourcentage d'entreprises ayant des innovations } \\
\text { protégées ou en voie de l'être }\end{array}$ & 47,07 & 33,33 & 1,95 \\
\hline $\begin{array}{l}\text { Pourcentage d'entreprises ayant } \\
\text { des collaborations d'affaires }\end{array}$ & 70,89 & 58,14 & $4,71 * *$ \\
\hline \multicolumn{4}{|l|}{ Organisation de la production des entreprises } \\
\hline $\begin{array}{l}\text { Pourcentage d'entreprises ayant une politique } \\
\text { visant à diminuer les délais de livraison }\end{array}$ & 74,87 & 65,15 & 2,35 \\
\hline $\begin{array}{l}\text { Pourcentage d'entreprises ayant une politique } \\
\text { visant à «gérer» les goulots de production }\end{array}$ & 66,16 & 56,25 & 2,06 \\
\hline $\begin{array}{l}\text { Pourcentage d'entreprises ayant une politique } \\
\text { visant à accroître la qualité des produits }\end{array}$ & 78,61 & 74,29 & 0,56 \\
\hline $\begin{array}{l}\text { Pourcentage d'entreprises ayant une politique } \\
\text { visant à réduire le temps de développement } \\
\text { des nouveaux produits }\end{array}$ & 48,97 & 41,18 & 0,67 \\
\hline $\begin{array}{l}\text { Pourcentage d'entreprises ayant une politique } \\
\text { visant à accroître la flexibilité des équipements }\end{array}$ & 44,30 & 42,62 & 0,05 \\
\hline $\begin{array}{l}\text { Pourcentage d'entreprises détenant } \\
\text { une norme reconnue de qualité }\end{array}$ & 67,12 & 57,50 & 2,38 \\
\hline
\end{tabular}

$* \mathrm{p}<0,10 ; * * \mathrm{p}<0,05 ; * * * \mathrm{p}<0,001$ 
Toutefois, les activités de recherche et développement plus importantes et le taux supérieur de détention d'un produit maison confirment que les entreprises exportatrices sont plus orientées vers l'innovation, comme le soutiennent Julien (2000), Sharmistha (1999) et Beamish et Munro (1986), et qu'elles peuvent ainsi avoir quelque chose d'unique à offrir à des clients étrangers ${ }^{11}$. Il pourrait d'ailleurs s'agir d'une condition pour pouvoir absorber une production plus coûteuse.

Selon notre deuxième hypothèse, les entreprises exportatrices sont plus productives que les autres, notamment grâce aux exigences de qualité et de compétitivité auxquelles elles doivent se conformer. Les résultats présentés au tableau 3 ne permettent pas de confirmer cette hypothèse en ce qui a trait à l'utilisation du capital physique (actifs) et du capital humain. Les exportateurs et les non-exportateurs ne présentent aucune différence significative, sauf pour le taux d'utilisation de l'actif total. Dans ce cas particulier, on constate que les exportateurs génèrent moins de ventes par dollar d'actifs, contrairement à ce qui avait été anticipé et cela n'est pas dû à des investissements plus substantiels dans les actifs de production dont les équipements, mais plutôt à des besoins supérieurs au niveau des actifs à

TABLEAU 3

Relations entre l'exportation, la productivité et la rentabilité des PME manufacturières

\begin{tabular}{|c|c|c|c|}
\hline & $\begin{array}{l}\text { Exportateurs } \\
(\mathrm{N}=237)\end{array}$ & $\begin{array}{l}\text { Non- } \\
\text { exportateurs } \\
(\mathrm{N}=86)\end{array}$ & $\begin{array}{c}\text { Test } \\
\text { statistique } \\
\text { (Probabilité) }\end{array}$ \\
\hline \multicolumn{4}{|l|}{ Mesures de productivité } \\
\hline Ventes / équipements de production & 6,99 & 7,29 & 0,65 \\
\hline Ventes / personnel administratif & $437955 \$$ & $401040 \$$ & 0,50 \\
\hline Ventes / actif total & 1,71 & 1,92 & $2,55 * *$ \\
\hline Bénéfice brut / personnel de production & $32533 \$$ & $28116 \$$ & 0,97 \\
\hline $\begin{array}{l}\text { Pourcentage des ventes des deux dernières } \\
\text { années attribuables à des nouveaux produits } \\
\text { ou à des produits modifiés }\end{array}$ & 40,23 & 31,29 & $2,88^{*}$ \\
\hline \multicolumn{4}{|l|}{ Mesures de rentabilité } \\
\hline Bénéfice brut / équipements de production & 1,47 & 1,48 & 0,13 \\
\hline Bénéfice brut/ventes (\%) & 22,87 & 20,14 & $2,48 * *$ \\
\hline Bénéfice net / ventes (\%) & 4,11 & 3,33 & $1,98 * *$ \\
\hline $\begin{array}{l}\text { Bénéfice disponible aux actionnaires / } \\
\text { fonds propres }(\%) \text { (ROE) }\end{array}$ & 17,31 & 15,47 & 0,85 \\
\hline Bénéfice d'exploitation / actif total (\%) (ROA) & 11,42 & 10,52 & 1,47 \\
\hline
\end{tabular}

$* \mathrm{p}<0,10 ; * * \mathrm{p}<0,05 ; * * * \mathrm{p}<0,001$.

11. Nous remercions l'un des évaluateurs pour son commentaire pertinent à ce sujet. 
court terme, soit les comptes clients et les stocks. Ces résultats confirmeraient ainsi les besoins supplémentaires en liquidités pour assurer le financement de ces éléments d'actif, comme nous l'avons relevé à la section 2.2.

En revanche, les exportateurs affichent un degré d'innovation supérieur aux non-exportateurs, comme l'indique le pourcentage de leurs ventes attribuables à des produits nouveaux ou modifiés, démontrant en cela une plus grande productivité des activités de R-D.

Notre troisième hypothèse pose que des coûts supérieurs, étant donné des activités administrative et de production plus complexes, devraient être absorbés par des prix plus élevés attribuables à des produits innovateurs, procurant ainsi une rentabilité équivalente chez les exportateurs et les non-exportateurs. Les résultats présentés au tableau 3 ne soutiennent pas entièrement notre hypothèse ; on voit en effet que les PME exportatrices affichent des marges bénéficiaires significativement plus élevées que les autres, alors que les taux de rendement réalisés sur leurs ressources (actifs et fonds propres) sont équivalents. Comme cela a été mentionné plus haut, les exportateurs possèdent plus d'actifs que les non-exportateurs de même taille et font ainsi moins de ventes par dollar d'actifs. Et bien qu'elles aient plus d'éléments d'actif à financer, elles n'utilisent pas plus le levier financier que les autres (résultat non présenté). C'est pourquoi le rendement des actifs et des fonds propres des deux groupes est identique même si les exportateurs font plus de profits.

Ces résultats pourraient démontrer l'importance des activités d'innovation pour les petits exportateurs, car elles permettent de récupérer une partie des coûts supplémentaires exigés par les ventes sur des marchés extérieurs. D'ailleurs, on constate que l'écart observé entre les marges bénéficiaires brutes des deux groupes s'est beaucoup atténué sur le plan des marges nettes comme le montre la valeur plus petite du test statistique ( 2,48 pour les marges brutes et 1,98 pour les marges nettes), confirmant l'ampleur des coûts associés aux activités administratives suscitées par l'exportation (frais de ventes et de gestion supplémentaires).

Le premier marché d'exportation des PME canadiennes est évidemment celui des États-Unis, étant donné sa proximité. La matrice des corrélations (tableau 4) montre une relation positive entre la taille et la propension à exporter. Toutefois, cette relation est moins forte lorsqu'on s'attarde à l'exportation outremer. Un test de corrélation de Pearson révèle en plus que cette relation n'est pas linéaire. Ces résultats confirment les nuances formulées par Sharmistha (1999) et Lefebvre et al. (1998) quant au rôle de la taille pour aider à prédire la propension à exporter des PME.

On note finalement que l'intensité des activités de R-D est plus fortement corrélée au pourcentage de ventes outre-mer qui sont également plus complexes que les ventes à proximité.

Revue internationale P.M.E., vol. 16, $\mathrm{n}^{\circ}$ 2, 2003

(C) 2003 - Presses de l'Université du Québec 
TABLEAU 4

Corrélation entre le taux de ventes à l'étranger et certaines caractéristiques générales des PME (probabilité)

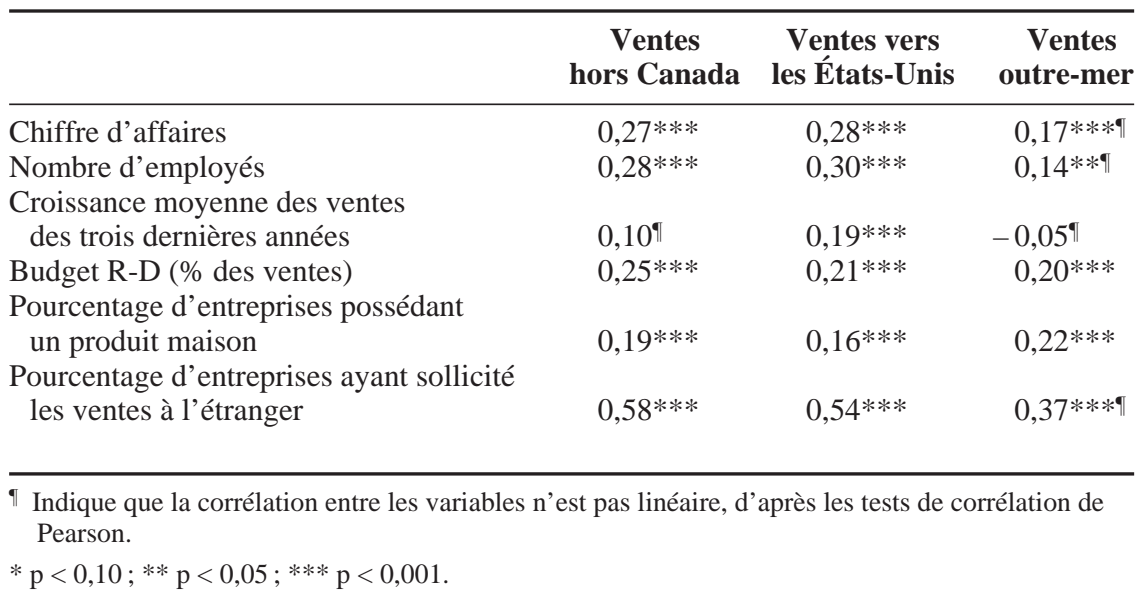

Selon notre quatrième hypothèse, le nombre de marchés d'exportation ou la diversité influence la rentabilité des entreprises. Les résultats du tableau 5 montrent effectivement que les entreprises qui exportent dans plus d'un marché (exportateur diversifié) affichent une marge bénéficiaire brute significativement supérieure aux autres. D'un autre côté, elles assument des frais de vente et d'administration plus élevés de telle sorte qu'elles ne présentent pas une meilleure marge bénéficiaire nette; ce sont des entreprises qui ont un chiffre d'affaires plus élevé et qui exportent une plus grande partie de leurs ventes, confirmant les résultats de Beamish et al. (1993).

Finalement, leurs activités d'exportation se font de façon plus organisée, car elles sollicitent en plus grande proportion les clients à l'étranger, plutôt que d'attendre les commandes internationales des clients potentiels. Notons par ailleurs que les autres variables organisationnelles et financières ne sont pas différentes (ces résultats ne sont pas présentés), notamment l'intensité des activités de R-D, l'utilisation des collaborations d'affaires et le taux de croissance moyen du chiffre d'affaires des trois dernières années.

Par ailleurs, nous avons voulu vérifier si la destination des exportations présentait certaines particularités. Les résultats du tableau 6 montrent que les exportateurs qui s'aventurent sur des marchés éloignés sont le fait d'entreprises plus grandes, plus matures, qui consacrent une plus grande partie de leurs activités à 
TABLEAU 5

Relations entre la diversité des exportations et la performance des PME

\begin{tabular}{lccc}
\hline & $\begin{array}{c}\text { Exportateur } \\
\text { diversifié } \\
(\mathrm{N}=82)\end{array}$ & $\begin{array}{c}\text { Exportateur } \\
\text { non diversifié } \\
(\mathrm{N}=154)\end{array}$ & $\begin{array}{c}\text { Test } \\
\text { statistique } \\
\text { (Probabilité) }\end{array}$ \\
\hline Chiffre d'affaires & $7,4 \mathrm{M} \$$ & $4,5 \mathrm{M} \$$ & $3,00^{* * *}$ \\
Âge des entreprises & 22 & 21 & 1,14 \\
Pourcentage d'exportation & 40,0 & 10,0 & $6,31^{* * *}$ \\
Pourcentage d'entreprises ayant un produit maison & 75,61 & 48,70 & $16,92 * * *$ \\
Pourcentage d'entreprises ayant sollicité & & & \\
$\quad$ les ventes à l'étranger & 91,46 & 64,29 & $22,15^{* * *}$ \\
Bénéfice brut/ventes & 26,12 & 21,65 & $3,00^{* * *}$ \\
Bénéfice net/ventes & 5,05 & 3,98 & 1,36 \\
\hline
\end{tabular}

$* \mathrm{p}<0,10 ; * * \mathrm{p}<0,05 ; * * * \mathrm{p}<0,001$.

l'exportation. Ces entreprises n'ont pas un taux d'innovation supérieur aux autres, mais ont développé en plus grande proportion leur propre produit maison et ont sollicité plus souvent les clients à l'étranger.

Toutefois, ces résultats sont contraires aux propositions de Lefebvre et al. (1998) voulant que la taille ne soit pas liée à la complexité des exportations. C'est le démarrage à l'exportation qui serait difficile et non le développement en intensité et en diversité qui, eux, influencent peu la rentabilité. On voit d'ailleurs que les PME qui exportent vers des marchés éloignés ont un degré d'exportation significativement plus important que celles qui se concentrent sur les marchés de proximité. Elles présentent également des caractéristiques communes aux entreprises qui ont une exportation diversifiée, comme l'illustre le tableau 5.

Finalement, Julien (2000) soutient que l'exportation chez les PME doit être analysée en tenant compte du degré d'organisation de l'entreprise pour répondre aux commandes extérieures et du fait que celles-ci soient sollicitées ou non. Nous avons tenu compte de cette hypothèse en effectuant des tests supplémentaires dont les résultats sont présentés au tableau 7. Il est ainsi possible de voir que les entreprises «proactives » à l'égard de l'exportation sont plus matures, exportent davantage et ont plus souvent leur propre produit maison. En revanche, mis à part la marge bénéficiaire brute, les indicateurs de rentabilité ne présentent pas d'écarts significatifs. 
TABLEAU 6

Relations entre la destination des exportations et la performance des PME

\begin{tabular}{lccc}
\hline & $\begin{array}{c}\text { États-Unis } \\
(\mathrm{N}=136)\end{array}$ & $\begin{array}{c}\text { Outre-mer } \\
(\mathrm{N}=101)\end{array}$ & $\begin{array}{c}\text { Test } \\
\text { statistique } \\
\text { (Probabilité) }\end{array}$ \\
\hline Chiffre d'affaires & $4,7 \mathrm{M} \$$ & $6,3 \mathrm{M} \$$ & $2,55^{* *}$ \\
Âge des entreprises & 19 & 23 & $1,97^{* *}$ \\
Pourcentage d'exportation & 10,00 & 35,00 & $4,93^{* * *}$ \\
Pourcentage des ventes des deux dernières années & & & \\
$\quad$ attribuables à des nouveaux produits & 40,98 & 39,39 & 0,10 \\
$\quad$ ou à des produits modifiés & 48,53 & 70,30 & $11,72^{* * *}$ \\
Pourcentage d'entreprises ayant un produit maison & & & \\
Pourcentage d'entreprises ayant sollicité & 66,18 & 83,17 & $8,82^{* * *}$ \\
$\quad$ des ventes à l'étranger & 21,65 & 24,90 & $2,28^{* *}$ \\
Bénéfice brut/ventes (\%) & 4,11 & 4,15 & 0,19 \\
Bénéfice net/ ventes (\%) & 12,07 & 11,05 & 0,86 \\
Bénéfice d'exploitation / actif total (\%) & & & \\
Bénéfice disponible aux actionnaires / & 17,97 & 16,36 & 0,39 \\
fonds propres $(\%)$ & & & \\
\hline
\end{tabular}

$* \mathrm{p}<0,10 ; * * \mathrm{p}<0,05 ; * * * \mathrm{p}<0,001$.

TABLEaU 7

Relations entre l'organisation de l'exportation, les caractéristiques et la performance financière des PME

\begin{tabular}{lccc}
\hline & $\begin{array}{c}\text { Exportateur } \\
\text { proactif } \\
(\mathrm{N}=174)\end{array}$ & $\begin{array}{c}\text { Exportateur } \\
\text { passif } \\
(\mathrm{N}=63)\end{array}$ & $\begin{array}{c}\text { Test } \\
\text { statistique } \\
\text { (Probabilité) }\end{array}$ \\
\hline Caractéristiques organisationnelles & & & \\
Chiffre d'affaires & $5,6 \mathrm{M} \$$ & $4,2 \mathrm{M} \$$ & 1,58 \\
Âge des entreprises & 22 & 19 & $1,91^{*}$ \\
Pourcentage d'exportation & 24,50 & 7,00 & $4,83^{* * *}$ \\
Pourcentage des ventes attribuables & & & \\
$\quad$ à des nouveaux produits dans les deux & & & \\
$\quad$ dernières années & 42,80 & 31,80 & $3,39^{*}$ \\
Pourcentage d'entreprises ayant un produit maison & 66,67 & 33,33 & $22,93^{* * *}$ \\
Exportation aux États-Unis (\%) & 17,00 & 5,00 & $3,85^{* *}$ \\
Exportation outre-mer (\%) & 0,20 & 0,00 & $3,22^{* * *}$ \\
Rentabilité & & & \\
Bénéfice brut/ventes (\%) & 24,23 & 21,36 & $1,87^{*}$ \\
Bénéfice net/ventes (\%) & 4,18 & 3,78 & 0,99 \\
Bénéfice d'exploitation / actif total (\%) & 11,89 & 10,67 & 0,99 \\
Bénéfice disponible aux actionnaires / & & & \\
$\quad$ fonds propres (\%) & 17,51 & 16,18 & 0,00 \\
\hline
\end{tabular}

$* \mathrm{p}<0,10 ; * * \mathrm{p}<0,05 ; * * * \mathrm{p}<0,001$. 


\section{Conclusion}

Dans cette étude, nous avons voulu vérifier la présence de relations entre l'exportation, le développement organisationnel et la situation financière des PME, afin de mieux comprendre, d'une part, pourquoi un faible pourcentage de petites et moyennes entreprises exportent et, d'autre part, pourquoi le taux d'exportation est relativement faible chez ces exportateurs.

L'étude d'un groupe de 323 PME manufacturières a conduit aux résultats suivants :

- Il existe une relation positive entre l'exportation et la taille des entreprises ;

- Les entreprises exportatrices ont un développement organisationnel semblable aux autres;

- Les entreprises exportatrices sont plus innovantes que les autres: elles réalisent plus de R-D, commercialisent un produit maison en plus grande proportion et un pourcentage supérieur de leurs ventes est attribuable à des produits nouveaux ou modifiés ;

- La diversité et l'éloignement des marchés sont liés à l'intensité des exportations, à la proactivité des entrepreneurs dans la recherche de clients à l'étranger et à la détention d'un produit maison par les entreprises ;

- L'exportation est liée à une rentabilité des ventes supérieure, mais ne procure pas des taux de rendement plus élevé (fonds propres et actifs), étant donné les besoins plus grands dans certains éléments d'actif (comptes clients et stocks).

Bien que les résultats précédents permettent de confirmer certaines hypothèses voulant qu'il existe des liens entre l'exportation des PME et certaines de leurs caractéristiques organisationnelles, de production et financières, nous ne pouvons pas établir de relations causales. Nous ne pouvons ainsi attribuer à leur implication sur les marchés étrangers la meilleure rentabilité des PME exportatrices ; les activités de R-D semblent jouer un rôle important dans ces résultats. Est-ce que ces activités sont liées au succès des PME à l'exportation et à leur capacité de trouver des clients pour acheter leur production ? Ou est-ce que ces activités sont imposées par des clients étrangers? Nous ne pouvons confirmer l'une ou l'autre des hypothèses.

À notre avis, vouloir encourager l'exportation chez les PME et accroître leur intervention sur les marchés internationaux implique qu'il faille développer leurs activités de R-D, que les entreprises doivent travailler à l'élaboration de leurs propres produits, qu'il est peut-être nécessaire de songer à diverses collaborations

Revue internationale P.M.E., vol. 16, nº 2, 2003 
d'affaires. Ces différentes actions pourraient favoriser la rentabilité des entreprises sur leur marché local, qui pourront ensuite songer à l'exportation sans mettre en péril leur survie ${ }^{12}$.

Nos résultats diffèrent de ceux d'autres auteurs, mettant ainsi en évidence la complexité du phénomène de l'exportation chez les PME, et le fait que d'autres études sont souhaitables pour mieux appréhender ses effets sur les entreprises. En particulier, nous n'avons pas tenu compte du secteur d'activités et du fait que certaines industries sont plus susceptibles de contribuer au développement d'activités internationales que d'autres, comme le résument bien Julien (1993). Dans ces conditions, il est possible que le dynamisme du secteur influence les stratégies et l'organisation des activités d'exportation et, en conséquence, les résultats financiers des PME exportatrices.

Par ailleurs, nous n'avons abordé qu'indirectement la problématique des besoins financiers des PME exportatrices. Il serait intéressant de voir dans quelle mesure le financement ou les liquidités peuvent être une contrainte à l'exportation des PME. À ce sujet, l'enquête de St-Pierre, Beaudoin et Desmarais (2001) a montré que le taux d'acceptation des demandes de marge de crédit était inversement lié au taux d'exportation des PME canadiennes. Or, nous avons relevé dans cette étude que les comptes clients et les stocks créaient des besoins supplémentaires de financement, lesquels sont habituellement assurés par une marge de crédit. Jusqu'à quel point les taux de refus élevés agissent comme un frein à l'exportation?

Il en est de même pour les activités de recherche et développement qui, à cause de l'incertitude de leurs résultats, suscitent d'importants problèmes de financement. Nos résultats ont mis en évidence le fait que l'exportation était fortement liée à l'innovation et à la R-D. La même enquête de St-Pierre et al. (2001) a fait ressortir les difficultés de financement qu'ont à surmonter les PME fortement engagées dans des activités de R-D. Ainsi, d'autres études pourraient examiner les stratégies que développent les PME pour exporter malgré l'engagement plutôt tiède des marchés financiers.

12. Il y a de plus en plus d'entreprises qui exportent dès le démarrage de leurs activités, étant ainsi presque invisibles sur le marché local. Nous n'avons pas abordé le cas de ces entreprises qui, dans les divers pays développés, demeurent bien plus des exceptions que des normes. Nous remercions toutefois l'un des évaluateurs pour nous avoir signalé que le développement des ventes à l'étranger n'est pas forcément conditionnel à la présence de l'entreprise sur le marché local. 


\section{Bibliographie}

BANK OF ENGLAND (1998), «Smaller exporters : a special report», janvier, disponible sur Internet : 〈http://www.bankofengland.co.uk/fin4smse.pdf >.

BANQUE DE DÉVELOPPEMENT DU CANADA (1997), «Beyond their small business : a survey », disponible sur Internet: 〈http://www.bdc.ca>.

BeAmish, P.W., R. CRAig et K. MCLelland (1993), «The performance characteristics of Canadian versus U.K. Exporters in small and medium sized firms », Management International Review, vol. 33, $\mathrm{n}^{\circ}$ 2, p. 121-137.

BEAmish, P.W. et H. MunRo (1986), «The export performance of small and mediumsized Canadian manufacturers », Canadian Journal of Administration Sciences, vol. $3, \mathrm{n}^{\circ} 1$, p. 29-40.

BiJMOLT, T.H.A. et P.S. ZWART (1994), «The impact of internal factors on the export success of Dutch small and medium-sized firms », Journal of Small Business Management, avril, p. 69-83.

BONACCORSI, A. (1992), «On the relationship between firm size and export intensity », Journal of International Business Studies, vol. 4, p. 605-635.

CALOF, J.L. (1993), «The impact of size on internationalization », Journal of Small Business Management, p. 60-69.

CANADIAN FEDERATION OF INDEPENDENT BUSINESS - CFIB/FÉDÉRATION CANADIENNE DE L'ENTREPRISE INDÉPENDANTE - FCEI (1997), « Market development survey highlights », disponible sur Internet: <http://www.cfib.ca/research/reports/mrktdev.asp>.

CAVUGSIL, S.T. (1984), «Organizational characteristics associated with export activity », Journal of Management Studies, vol. 21, no 1, 4-22.

ClARKE, W. (2000), «The use of foreign languages by Irish exporters », Europan Journal of Marketing, vol. 34, n 1, p. 80-90.

COOPER, S. et I. NYBORG (1998), «The financing and information needs of smaller exporters », Bank of England Quarterly Bulletin, mai, p. 166-172.

De Toni, A. et G. NAssimbeni (2001), «The export propensity of small firms: a comparison of organisational and operational management levers in exporting and non-exporting units », International Journal of Entrepreneurial Behavior \& Research, vol. 7, no 4, p. 132-147.

HeUNKS, F.J. (1998), «Innovation, creativity and success », Small Business Economics, vol. 10, p. 263-272.

InDUSTRY CANADA (1997), Small Business Quarterly, disponible sur Internet : <http:// strategis.ic.gc.ca>.

JULIEN, P.-A. (1993), « Globalisation de l'économie et PME», Communication présentée au Congrès international francophone de la PME (CIFPME), Carthage, Tunisie. Actes du colloque.

JULIEN, P.-A. (2000), L'entrepreneuriat au Québec: pour une révolution tranquille entrepreneuriale 1980-2005, Montréal, Les Éditions Transcontinental, 399 p.

Revue internationale P.M.E., vol. 16, $\mathrm{n}^{\circ}$ 2, 2003

(C) 2003 - Presses de l'Université du Québec 
LEE, D.J. et J.I. JANG (1998), «The role of relational exchange between exporters and importers : evidence from small and medium-sized Australian exporters », Journal of Small Business Management, vol. 36, $\mathrm{n}^{\circ}$ 4, p. 12-23.

Lefebvre, E., L.A. LefebVRE et M. Bourgault (1998), «R\&D related capabilities as determinants of export performance », Small Business Economics, vol. 10, p. 365-377.

LÉO, P.Y. (2000), «Les PME et les couples produits-services à l'exportation», Revue internationale PME, vol. 13, no 2, p. 45-73.

LÉONIDOU, L.C. et A.S. ADAMS-FLOROU (1997), «Types and sources of export information : insights from small business », International Small Business Journal, vol. 17, $\mathrm{n}^{\circ}$ 3, p. 30-48.

MATHESON, D. (2000), «Achieving performance excellence », New Zealand Management, vol. $47, \mathrm{n}^{\circ} 1$, p. 54-55.

Mehran, J. et A.H. MoINI (1999), «Firms' export behavior», American Business Review, vol. $17, \mathrm{n}^{\circ} 1$, p. $86-93$.

MoINI, A.H. (1995), « An inquiry into successful exporting : an empirical investigation using a three-stage model », Journal of Small Business Management, juillet, p. 9-25.

REYNOLDS, P.D. (1997), «New and small firms in expanding markets », Small Business Economics, vol. 9, n 1, p. 79-84.

SAMUEls, J., S. GREENFIELD et H. MPUKu (1992), «Exporting and the smaller firm », International Small Business Journal, vol. 10, nº 2, p. 24-36.

SHARMisthA, B.-S. (1999), «The small and medium sized exporters' problems: an empirical analysis of Canadian manufacturers », Regional Studies, vol. 33, no 3, p. 231-245.

STATISTIQUE CANADA (1994-1996), «Interprovincial Trade in Canada », disponible sur Internet : <http://www.statcan.ca>.

St-Pierre, J., R. BeAudoin et M. Desmarais (2001), Le financement des PME canadiennes: satisfaction, accès, connaissance et besoins, Rapport de recherche pour Industrie Canada, décembre, 400 p. Disponible sur Internet: <http://strategis. ic.gc.ca/SSGF/rd00563f.html>.

StumP, R.L., G.A. Athaide et C.N. AxinN (1998), « The contingent effect of the dimensions of export commitment on exporting financial performance: an empirical examination », Journal of Global Marketing, vol. 12, $\mathrm{n}^{\mathrm{o}}$ 1, p. 7-25.

WALTERS, P.G.P. et S. SAMIEE (1990), «A model for assessing performance in small U.S. exporting firms », Entrepreneurship Theory and Practice, vol. 15, p. 33-50.

WeSTHEAD, P. (1995), «Exporting and non-exporting small firms in Great Britain », International Journal of Entrepreneurial Behaviour \& Research, vol. 1, nº 2, p. 6-36.

WolfF, J.A. et T.L. PETT (2000), «Internationalization of small firms : an examination of export competitive patterns, firm size, and export performance », Journal of Small Business Management, vol. 38, $\mathrm{n}^{\circ}$ 2, p. 34-47.

Zahra S.A., D.O. NeUbaum et M. HuSE (1997), « The effect of the environment on export performance among telecommunications new ventures », Entrepreneurship Theory and Practice, vol. 22, n ${ }^{\circ}$ 1, p. 25-46.

Revue internationale P.M.E., vol. 16, $n^{\circ}$ 2, 2003 